\title{
Ulnar deviation is not always rheumatoid
}

\author{
M Zuber, C Braun, M Pfreundschuh, W Püschel
}

Internal Medicine I, University Medical Centre, Homburg, Germany

M Zuber

M Pfreundschuh

Department of Traumatology, Hand and Reconstructive Surgery, University Medical Centre, Homburg, Germany C Braun

Department of Pathology, University Medical Centre, Homburg, Germany W Püschel

Correspondence to: Margit Zuber, University Medical Centre, Internal Medicine I, D66421 Homburg, Germany

Accepted for publication 18 July 1996

\section{Case report}

A 46 year old right handed white female presented in January 1995 with a two year history of ulnar deviation of the metacarpophalangeal (MCP) joints on both hands. During the past couple of months the extent of ulnar deviation had increased rapidly and swan neck formation of the proximal interphalangeal joints of the fourth and fifth finger of her left hand had developed. There were synovial swellings of the second to fifth MCP joints on both hands, which were much more pronounced on the left side. Eventually the patient could not open her left hand because of incomplete extension of the MCP joints (fig 1A). She was concerned because of the fixed flexion deformity of her MCP joints which caused inability to use her left hand properly. She did not complain of pain.

The MCP joints of the patient were swollen but no signs of inflammation were present. The joint swelling was firm. No redness, tenderness, or warmth was present. There was tendon crepitus elicited on the extensor heads. No swelling of any other joints was visible. The patient denied morning stiffness, pain, and symptoms of past arthritis of any joint. There were no rheumatoid nodules and $x$ ray showed no erosive signs of arthritis. The patient had a history of breast carcinoma of the left side which was cured by surgery in 1992. Apart from the deformity she felt well.

Erythrocyte sedimentation rate, $\mathrm{C}$ reactive protein, protein electrophoresis, full blood count, and blood chemistry were normal. Rheumatoid factor and antinuclear antibody tests were negative.

The suspected diagnosis of rheumatoid arthritis could not be established. A connective tissue disease such as systemic lupus erythematosus was not present; therefore the symptoms could not be interpreted as Jaccoud's arthropathy. The patient did not have nail pitting and skin lesions suggesting an inflammatory arthritis such as psoriatic arthritis. There were no clues for the underlying cause of the ulnar deviation.

In order to restore the proper function of the hand, synovectomy of the second to fifth MCP joints and reconstruction of the extensor hood of the left hand was performed. During the operation a hypertrophic red-brown synovium resembling villonodular synovitis was visible (fig 1B). This had caused the extensive weakering of the extensor hood and ulnar dislocation of the extensor tendons. Synovectomy and reconstruction of the extensor hood was performed.

Pathological evaluation of the synovial tissue showed villous and nodular structures, with infiltration of many foamy histiocytes, a few giant cells, and scattered lymphocytes in a fibrous stroma, as well as deposits of haemosiderin, a feature which is characteristic of pigmented villonodular synovitis (fig $2 \mathrm{~A}$ and B).

One year after surgery the patient is well. No recurrence of synovitis had occurred up to now.

\section{Discussion}

This patient suffered from pigmented villonodular synovitis (PVNS), which is an uncommon disorder. ${ }^{1}$ In a large study ${ }^{2}$ the incidence of this disease was 1.8 per million population in patients undergoing surgery. It is more common in females than in males. Its highest incidence is in females in the third and fourth decade. It presents as slowly progressive benign proliferation of synovial tissue. The term was introduced in 1941 by Jaffe et al, ${ }^{3}$ who also described the salient histological features: deposition of haemosiderin and infiltration of histiocytes and giant cells in a fibrous stroma within the synovium of tendon sheaths and large joints.

PVNS presents in three different clinical forms. ${ }^{3}$ Pigmented villonodular tenosynovitis (PVTS), also referred to as giant cell tumour of tendon sheaths, ${ }^{4}$ is the first and most common expression of what is believed to be a disease spectrum of three basic types of clinical presentation. This isolated lesion is usually painless, slowly progressive, and may cause erosions of adjacent bone. The hand, especially the fingers, is the most common site. The lesion is identical to localised PVNS and histologically identical to diffuse PVNS. The second presentation is diffuse intra-articular PVNS. ${ }^{5}$ This form has attracted the most attention, and serves as the prototype for aetiological investigations. The knee is the most commonly involved joint, followed by the hip and ankle. Infrequently, this diffuse form presents in the hand, shoulder, wrist, and vertebrae. Progression is slow with initially intermittent and later persisting joint swelling and pain. Diffuse PVNS closely resembles the 

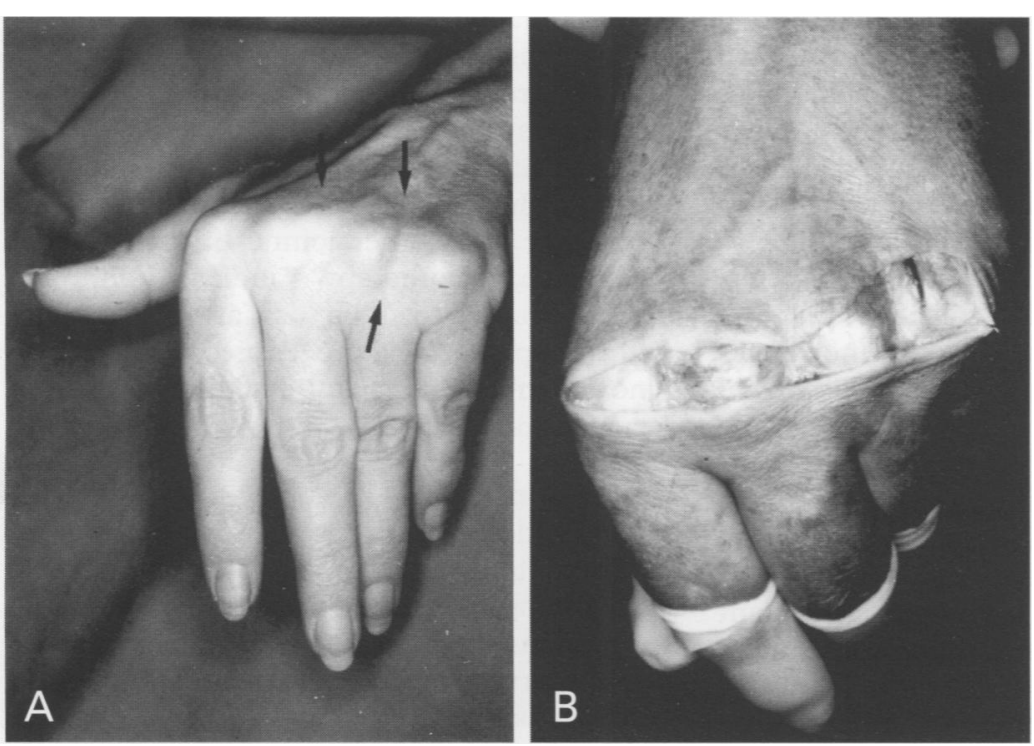

Figure 1 Left hand of the patient with the fixed flexion deformity and ulnar deviation of the metacarpophalangeal joints. (A) Dislocation of the extensor tendons is marked; (B) Intraoperative site.

chronic synovitis of rheumatoid arthritis. The third and least common presentation ${ }^{7}$ is localised pedunculated villonodular synovitis (LPVS). The knee is the joint most usually
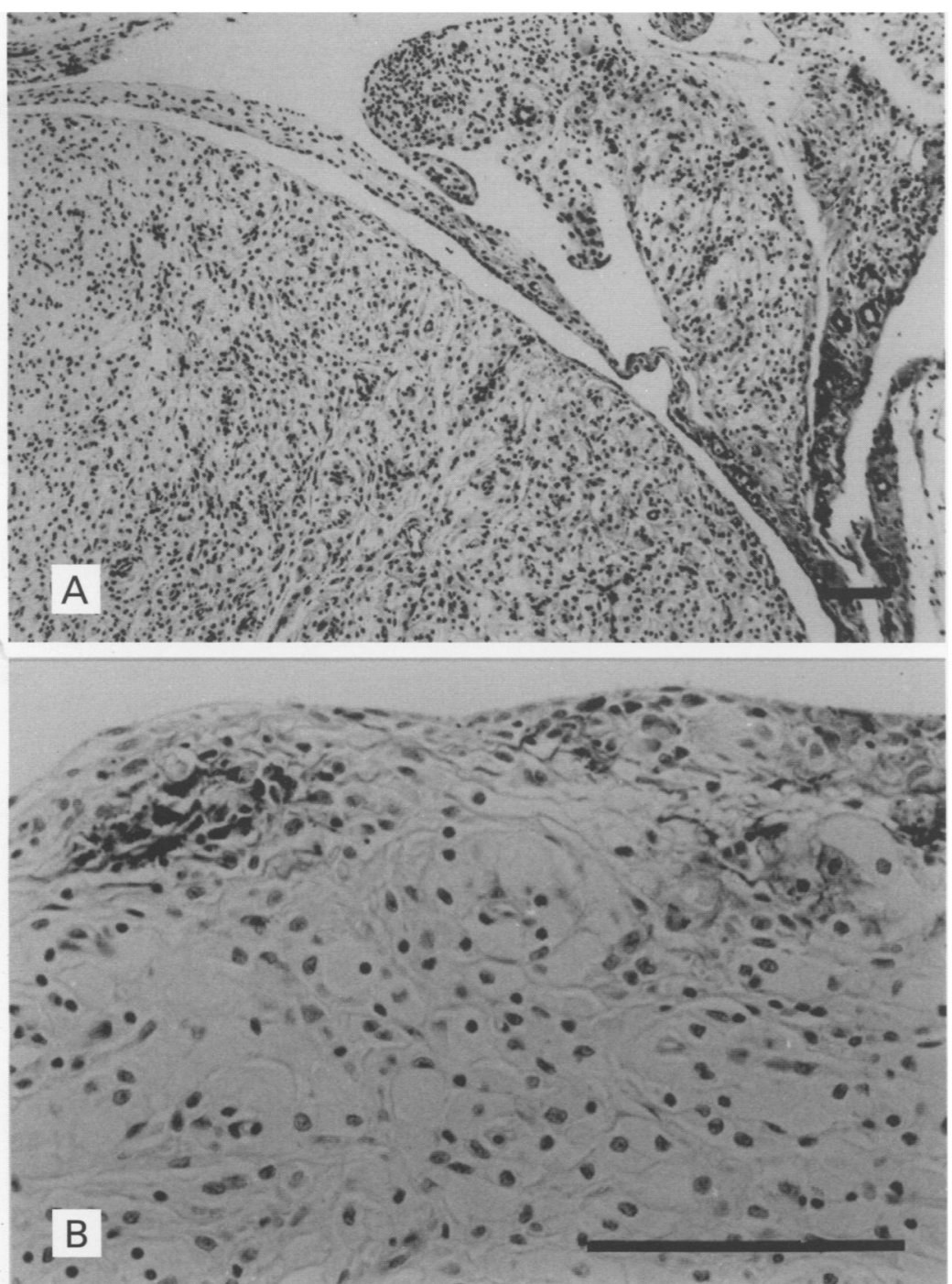

Figure 2 Pigmented villonodular synovitis. (A) Characteristic microscopical appearance. Bar: $30 \mu \mathrm{m}$. (B) Infiltration of foamy histiocytes, giant cells and scattered lymphocytes. (haematoxylin-eosin). Bar: $30 \mu \mathrm{m}$. involved. The lesion is initially thought to be a torn meniscus or a loose body.

The patient presented here belongs to the second group of diffuse or multiple regional intra-articular PVNS. Localisation in the MCP joints is very unusual. Synovial swelling caused by tumorous tissue is responsible for the ulnar deviation by the same mechanisms as proposed in rheumatoid arthritis or systemic lupus erythematosus. ${ }^{8}$ Chronic synovial inflammation and proliferation causes stretching of radial collateral ligament and slippage of extensor carpi ulnaris tendon in a lateral-volar direction and these forces cause volar subluxation and radial rotation of the carpus. This results in ulnar deviation of the MCP joints. Alternatively, ulnar deviation may be caused by stretching of volar supports of flexor tendons at the MCP joints, with a resultant pull in an ulnar direction during flexion. Ulnar deviation of MCP joints, together with a predominance of ulnar deviating forces during power grip of fine work, causes slippage of the extensor tendons in an ulnar direction secondary to ulnar subluxation and MCP joint capsular stretching. This increases ulnar deviation of the MCP joints.

PVNS belongs-together with five other conditions - to the group of benign tumorous conditions of articular structures (table) ${ }^{9}$ which should be kept in mind if unusual presentations of symptoms need to be evaluated. Synovial chondromatosis arises from focal metaplasia of subsynovial tissue, producing nodules of normal appearing cartilage, bursa, tendon sheath, joint capsule, or para-articular connective tissue. These nodules become pedunculated and often detach from the synovium, when they grow as viable lose bodies within the joint cavity. These cartilage nodules may calcify or ossify, leading to the term osteochondromatosis. The joint most often involved is the knee, followed by the hip, the elbow, and the shoulder. Patients may have pain, swelling, and limitation of joint motion, or they may be asymptomatic, with the lesion discovered by accident. Examination of the joint often reveals the presence of loose bodies and increased amounts of synovial fluid. Vascular malformations such as haemangioma most often affect the synovial membrane of the knee. They occur most commonly in adolescents or young adults, many of whom have had symptoms since childhood suggesting a congenital vascular malformation. The affected joint is periodically painful and swollen and often contains blood or bloody fluid. A haemangioma within the joint can be localised in the form of a dark, grape-like mass, or it can be diffuse. It is especially difficult to differentiate diffuse haemangioma from pigmented villonodular synovitis, and histological evaluation is necessary. Fatty growths, such as intra-articular lipomas, are rare and favour the knee, but lipomas are also found in the tendon sheaths of the hand, wrist, feet, and ankles. Extensor tendons appear to be affected more often than flexor tendons, and involvement may be bilateral. Fibromata are often located on tendon sheaths. They consist of firm, lobulated tumours attached to the ten- 
don or the tendon sheath. The majority of lesions occur in the hand and wrist. Intra-articular fibromata are rare. The nodules are composed of fibroblasts and dense collagen. The lesion is benign, but recurrences are frequent following local excision.

Other differential diagnoses include the second stage of haemophilic arthropathy, which is characterised by a chronic proliferative synovitis in response to repeated haemorrhages within the joint. Pathologically, the synovial lining cells proliferate, as does the connective tissue in the joint capsule. Foreign body granuloma is another differential diagnosis. Monarticular synovitis, tenosynovitis, or other soft tissue reactions can be induced by the penetration of a foreign body such as a plant thorn, wood splinter, or sea urchin spine into a joint, tendon sheath, or periarticular soft tissues. Tuberculous arthritis should always be considered as a possible cause of an unexplained monarticular arthritis, although it is relatively uncommon in western countries. Synovial tissue histology and culture for the presence of acid fast bacilli are the basis of diagnosis.

Our patient was treated with synovectomy and no recurrence had occurred up to one and a half years later. In a retrospective analysis of 99 selected patients with PVNS of the knee, hip, elbow, or shoulder, ${ }^{5}$ patients were followed longitudinally for a mean of 13.5 years after the index operative procedure at the authors' institution. During this time 25 patients had a recurrence. Using the Kaplan-Meier analyses,

Benign tumorous conditions of articular structures

Pigmented villonodular synovitis

Synovial chondromatosis

Cartilaginous metaplasias of the synovial tissues

Vascular malformations

Fatty growths

Fibroma the cumulative probability of being continuously recurrence-free at 25 years was $65 \%$. Surgical procedures that do not remove all the diseased synovium are more likely to be followed by recurrence. The knee joint has a significantly greater chance of having a PVNS recurrence than other joints. In the event of recurrence, radiotherapy is indicated although the response to this is also uncertain. Arthroscopic surgical procedures and radiation synovectomy ${ }^{10}$ hold promise in the treatment of recurrent disease.

\section{The lesson}

- Ulnar deviation is not always a result of rheumatoid arthritis, systemic lupus erythematosus, or psoriatic arthritis.

- Synovitis may be due to benign tumorous disorders such as pigmented villonodular synovitis.

- Histological evaluation is a very important tool for establishing a diagnosis.

1 Bentley G, McAuiffe T. Pigmented villonodular synovitis. Ann Rheum Dis 1990;49:210-11.

2 Myers BW, Masi AT, Feigenbaum SL. Pigmented villonocular synovitis and tenosynovitis. A clinical epidemiological study of 166 cases and literature revies. Medicine (Baltimore) 1980;59:223-38.

3 Jaffe HI, Lichtenstein L, Sutro CJ. Pigmented villonodular synovitis, bursitis and tenosynovitis. Arch Pathol 1941; 31:731-65.

4 Jones FE, Soule EN, Coventry MD. Fibrous xanthoma of synovium (giant cell tumor of tendon sheath, pigmented nynovium (giant cell tumor of tendon sheath, pigmented 5 Schwartz HS, Unni KK, Pritchard DJ. Pigmented villonodular synovitis, a retrospective review of affected joints. odular synovitis, a retrospecti
Clin Orthop 1989;247:243-55.

6 Flandry F, Hughston JC. Current concepts review, pigmented villonodular synovitis. $\mathcal{F}$ Bone foint Surg Am 1987; 69A:942-9.

7 Fraire AE, Fechner AE. Intra-articular localized nodula synovitis of the knee. Arch Pathol 1972;93:473-6.

8 Harris ED. The clinical features of rheumatoid arthritis. In: Kelley WN, Harris ED, Ruddy S, Sledge CB, eds. Textbook of rheumatology, 3rd ed, vol 1. Philadelphia: WB Saunders, 1989:959.

9 Cohen AS, Canoso JJ. Tumors of joints and related structures. In: McCarthy DJ, ed. Arthritis and allied condistructures. In: McCarthy DJ, ed. Arthritis and allied condi-
tions. Philadelphia: Lea \& Fiebinger, 1989:1492-508.

10 Wiss DA. Recurrent villonnocular synovitis of the knee. Successful treatment with yttrium-90. Clin Orthop 1982; 169:139-44. 